\title{
Workshop: physiology and tolerance of LDCs
}

\author{
John Cummings ${ }^{1}$, Adam Lee ${ }^{2}$ and David Storey ${ }^{2}$ \\ ${ }^{1}$ MRC Microbiology and Gut Biology Group, Ninewells Hospital and Medical School, Dundee, DD1 9SY, Scotland, UK \\ ${ }^{2}$ Nutritional Biosciences Group, Division of Biological Sciences, School of Environment and Life Sciences, The University of \\ Salford, Greater Manchester, M5 4WT, UK
}

Gastrointestinal symptoms

Although it is accepted that consumption of low-digestible carbohydrates (LDCs) can have undesirable gastrointestinal effects in some individuals, few tolerance studies are comparable in terms of symptom definition or the methodology adopted. This workshop considered the main gastrointestinal symptoms that can occur following consumption of LDCs and if current symptom definitions were wholly appropriate. The study protocols that should be adopted to investigate the gastrointestinal (GI) tolerance of LDCs were discussed and what affects tolerance, such as host factors and types of LDC ingested. The workshop debated if the occurrence of GI symptoms following consumption of LDCs outweighed their perceived functional benefits.

\section{Possible gastrointestinal symptoms following consumption of LDCs}

GI symptoms following consumption of LDCs arise from their osmotic effect in the GI tract and their fermentation by colonic bacteria. These effects may lead to changes in bowel habit and uncomfortable abdominal symptoms due to intestinal gas. The main symptoms that arise from ingestion of LDCs may be generally classified as: (a) changes in bowel habit; (b) painful sensations; (c) gaseous symptoms; (d) other symptoms such as nausea (Table 1). The workshop agreed that GI symptoms following consumption of LDCs by individuals were subjective in nature and therefore difficult to assess. Wind, diarrhoea, borborygmi and bloating are experienced by many individuals unrelated to LDC dietary intake and may be considered as a normal occurrence by them. The workshop therefore agreed that the occurrence of GI sensations or changes in bowel habit over and above what individuals perceive as 'normal' should be used to define GI symptoms.

Because GI symptoms are difficult to measure the workshop agreed that double-blind, controlled cross-over studies should be used to assess the GI responses of individuals following consumption of LDCs. Furthermore, any association between different symptoms and the
Table 1. Potential gastrointestinal symptoms following consumption of LDCs

\begin{tabular}{ll}
\hline Changes in bowel habit & $\begin{array}{l}\text { Diarrhoea } \\
\text { Increased laxation }\end{array}$ \\
Painful symptoms* & Abdominal colic (cramps, stomach ache) \\
Gaseous symptoms & $\begin{array}{l}\text { Abdominal bloating } \\
\text { Abdominal noise (borborygmi) }\end{array}$ \\
& $\begin{array}{l}\text { Flatulence } \\
\text { Other symptoms }\end{array}$ \\
& Nausea \\
& Loss of appetite \\
& Thirst \\
& Headache
\end{tabular}

* Symptoms associated with excretion of stools may be reduced in severity.

occurrence of more than one symptom, i.e. multiple symptoms should be considered.

\section{Bowel habit}

There is great variability amongst individuals in bowel habit. The normal range is from three bowel movements per week to three per day, with a modal frequency of one per day. Changes in bowel habit may be assessed by a variety of means. Stool collection provides useful data in terms of stool weight and consistency but is difficult to achieve except in controlled diet studies and is not practical in community-based investigations. In community studies, bowel movement frequency, consistency, urgency and

Table 2. Potential health benefits of LDCs

Reduced cariogenicity

Reduced energy intake

Increased satiety

Glycaemic control

Reduced lipid absorption

Reduced serum LDL

Prebiotic effects

Antineoplastic effects

Increased laxation

Improved calcium absorption

Reduced diffusion of ammonia into portal blood

\footnotetext{
* Corresponding author: John Cummings, fax +44 (0) 1382633 952, email j.h.cummings@dundee.ac.uk
} 
Table 3. Possible factors that may effect tolerance of LDCs

\begin{tabular}{|c|c|c|c|}
\hline Host factors & Type of LDC & Dietary factors & Drug treatment \\
\hline $\begin{array}{l}\text { Composition of colonic flora } \\
\text { Psyche } \\
\text { Age } \\
\text { Gender } \\
\text { Menstrual cycle } \\
\text { Pregnancy } \\
\text { Diseases } \\
\text { Irritable bowel syndrome } \\
\text { Coeliac disease } \\
\text { Diabetes } \\
\text { Inflamatory bowel disease } \\
\text { Gastrointestinal transit time } \\
\text { Enzyme activity } \\
\text { Visceral sensitivity }\end{array}$ & $\begin{array}{l}\text { Chemical characteristics } \\
\text { Molecular weight } \\
\text { Sugar composition } \\
\text { Degree of polymerisation/branching } \\
\text { Resistance to upper intestinal hydrolysis } \\
\text { Degree of upper intestinal absorption } \\
\text { Fermentability of LDC in colon }\end{array}$ & $\begin{array}{l}\text { Consumption pattern } \\
\text { Amount ingested } \\
\text { Frequency } \\
\text { Consumption with liquids/solids } \\
\text { Other LDCs in diet } \\
\text { Naturally occurring LDCs } \\
\text { LDCs added as ingredients to foods }\end{array}$ & Antibiotics \\
\hline
\end{tabular}

other observations such as stool colour and odour may be recorded in diaries by study participants. Such data are useful in determining changes in bowel habit following consumption of LDCs.

\section{Intestinal gas}

Colonic fermentation of LDCs may lead to a rapid build up of intestinal gas and the symptoms of abdominal colic, bloating, abdominal noise (borborygmi) and flatulence. The workshop considered that each of these symptoms could be subjectively measured in terms of frequency, severity and duration by use of subject diaries and interview. Measurement of abdominal girth was also identified as a useful index of abdominal bloating. Because gases are absorbed across the colonic mucosa and excreted via the lungs breath hydrogen and methane analyses were seen as useful techniques in the assessment of gastrointestinal fermentation following consumption of LDCs.

\section{Benefits and disadvantages of LDC consumption}

The workshop considered the potential health benefits following consumption of different LDCs compared to the potential disadvantages in terms of GI symptoms. The workshop broadly agreed that the benefits of LDC consumption outweighed their disadvantages (Table 2). The workshop agreed that GI symptoms following consumption of LDCs were often transient compared to the lasting benefits derived from their consumption and those individuals that find themselves sensitive to the effects of LDC ingestion can reduce or stop their intake with no further effects. However, LDCs in different product applications have different potential health benefits, for example polyols in sugar-free products are non-cariogenic whereas fructo-oligosaccharides are promoted as more prebiotic in nature.

\section{Factors that affect tolerance}

The workshop discussed some of the factors that affect tolerance of LDCs in view of the presentations made by Drs Marteau and Livesey (p.S17-S21 and S7-S16). It is apparent that GI tolerance does not depend solely on the type of LDC or the dose ingested but on many other factors. These are summarised in Table 3. However, the effect on GI tolerance of LDC consumption pattern, naturally occurring LDCs in the diet and interactions with other dietary components requires further research (Cummings et al.).

\section{Reference}

Cummings JH, MacFarlane GT \& Englyst HN Prebiotic digestion and fermentation. American Journal of Clinical Nutrition (In press). 